\title{
Flip-Over and Oscillation Phenomena of Fiber Orientation Ellipsoids in Bi-Disperse Suspension Flows
}

\author{
CHIBA Kunji ${ }^{\text {a) }}$ and Francisco CHINESTA ${ }^{\text {b) }}$

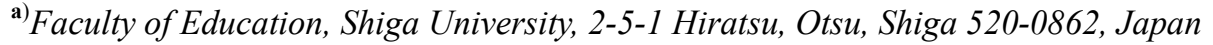 \\ b) LMSP: UMR CNRS - ENSAM - ESEM, 151 Boulevard de l'Hôpital, F-75013 Paris, France
}

Received January 24, 2005; Accepted for Publication February 15, 2005

\begin{abstract}
Fiber suspension flow and fiber orientation through a parallel plate channel were numerically simulated for mono- and bi-disperse fiber suspensions in order to study the mechanism of the evolution of fiber orientation distribution. In the present simulations, fiber-fiber interaction was not taken into account.

For suspensions including small aspect-ratio fibers, flip-over or oscillation phenomenon of the orientation ellipsoid was predicted. For the $r_{a}=10+40$ (bi-dispersion including fibers with aspect-ratio $r_{a}=10$ and $\left.r_{a}=40\right)$ and $r_{a}=10+100$ suspensions, fibers with $r_{a}=40$ and $r_{a}=100$ almost completely aligned along the channel wall, however these fibers had little influence on the evolution of the orientation ellipsoids for the bi-disperse suspensions because the number of fibers was much lower when the same fiber parameters were assumed for both small and large aspect-ratio fibers. In contrast fibers with $r_{a}=20$ could affect the evolution of the orientation ellipsoids for the bi-dispersion with $r_{a}=10+20$. It could be found that the phenomenon taking place, flip-over or oscillation, depends on the initial orientation distribution of fibers, the number of fibers of each aspect-ratio for bi-dispersion and the flow kinematics of fiber suspension.
\end{abstract}

Keywords: evolution of orientation ellipsoid, bi-disperse fiber suspension, flip-over, oscillation phenomenon, statistical scheme

\section{Introduction}

Fiber suspension can often exhibit anisotropy due to flow-induced fiber alignment. The addition of short fibers to a Newtonian liquid can, therefore, drastically change the flow kinematics even at very low concentrations. Furthermore, the fiber orientation state strongly affects the physical properties of short-fiber composites and the characterization of fiber orientation distribution becomes much more significant in current processing of high quality composite materials. For the above reasons, the importance of the coupling flow kinematics with fiber orientation has been recognized during the last two decades [1-10].

In a previous paper, Chiba and Chinesta [11] simulated the 2-D suspension flows involving both quasi infinite and small aspect-ratio $\left(r_{a}=10\right)$ fibers in a Newtonian fluid through a parallel plate channel by coupling flow kinematics with 3-D fiber orientation distribution. As a result, the flip-over phenomenon had a strong influence on the flow kinematics and fiber orientations. It is well known that fibers with small aspect-ratio frequently and periodically flip over when fiber-fiber interaction does not exist. Flip-over is a phenomenon related to the rapid rotation of a slender particle that occurs when it is aligned in the perpendicular direction to the flow.

The just referred study on fiber suspension flows focused on flow kinematics and fiber orientation for mono-disperse fiber suspensions. In the processing of fiber composites, however, length of suspended fibers is always distributed because of fiber breakage during processing [12]. Thus, the analysis of the effect of fiber length distribution on the flow kinematics is necessary to model multi-disperse fiber suspensions.

In our recent work [13], the development of flow kinematics and fiber orientation distribution was computed in multi-disperse suspension flow through a parallel plate channel and their predictions were compared with those obtained for mono- and bi-disperse suspensions. For suspensions including small aspect-ratio fibers, flip-over or oscillation phenomenon of the orientation ellipsoid (schematic illustration of fiber orientation distribution) caused the wavy patterns of the centerline velocity profile and streamlines as well as the rapid and complex variation of the shear stress and the normal stress difference near the channel wall.

In this work, coupled flow kinematics and 3-D fiber orientation distribution have been simulated, then the mechanism of the flip-over or oscillation phenomenon of the 
orientation ellipsoid for mono- and bi-disperse suspensions has been examined in detail, when both the fiber-fiber and fiber-wall interactions are neglected, using a statistical scheme: we evaluate the orientations of a large number of fibers with specified initial angles by solving the Jeffery equation along the streamlines. Fiber suspensions considered in the present work consist of rigid fiber suspensions in a Newtonian liquid. A parabolic velocity profile and an isotropic orientation state being assumed at the inlet of a parallel plate channel, the development of the 3-D fiber orientation has been analyzed.

\section{Numerical Solution Procedure for Suspension Flow}

We computed the 2-D flows (taking place in the $x-y$ plane) of suspensions with mono- and bi-dispersed fibers in a Newtonian fluid by coupling the flow kinematics with the 3-D fiber orientation distributions to study the evolution of fiber orientation distribution in a fiber suspension flow through a parallel plate channel. Detailed description of the governing equations, statistical scheme and solution procedure for mono- and bi-disperse fiber suspensions can be found in our former papers [11] and [13], respectively. The governing equations, therefore, are not given here and the statistical scheme and the solution procedure will be briefly introduced.

\subsection{Statistical scheme}

The evolution equation of the vector $p$ defining the orientation of a fiber immersed in a Newtonian homogeneous flow can be described by the Jeffery equation,

$$
\frac{d \underline{p}}{d t}=\underline{\Omega} \underline{p}+k\left(\underline{D}-\underline{p^{T}} \underline{\underline{D}} \underline{p}\right) \underline{p},
$$

where $\underline{\underline{\Omega}}$ is the vorticity tensor and $\underline{\underline{D}}$ is the deformation-rate tensor. The parameter $k$ is given $\overline{\bar{b}}$ y

$$
k=\left(r_{a}^{2}-1\right) /\left(r_{a}^{2}+1\right),
$$

where $r_{a}$ is the fiber aspect-ratio.

The second-order orientation tensor $a$ defined by Eq.(3) is introduced, which is a suitable and concise way for describing the orientation distribution of fibers [14], where $\psi(p)$ is the orientation distribution function. Then, the orientation state can be illustrated using the usual orientation ellipsoid (e.g., see Fig.1). The eigenvalues and eigenvectors of $a$ give the lengths and directions of the three major axes of the ellipsoid, and also the eigenvalues indicate the degree or intensity of the orientation along these directions.

$$
\underline{\underline{a}}=\oint \underline{p} \otimes \underline{p} \psi(\underline{p}) d \underline{p} .
$$

We evaluated the orientation of a large number of fibers $N$ with specified initial angles by solving the Jeffery equation (1) along the streamlines using a 4th-order Runge-Kutta method. The time step used in the discretisation of the Jeffery equation depends on the streamline considered, and its maximum value is fixed to 0.05 in the wall neighborhood. Then, the components of $a$ could be calculated along the streamlines by Eq. (4) for the 3 -D orientation of fibers.

$$
\begin{aligned}
& a_{11}=\sum_{i=1}^{N} \sin ^{2} \theta_{i} \cos ^{2} \beta_{i} / N, a_{12}=\sum_{i=1}^{N} \sin ^{2} \theta_{i} \cos \beta_{i} \sin \beta_{i} / N, \\
& a_{13}=\sum_{i=1}^{N} \sin \theta_{i} \cos \theta_{i} \cos \beta_{i} / N, a_{22}=\sum_{i=1}^{N} \sin ^{2} \theta_{i} \sin ^{2} \beta_{i} / N,
\end{aligned}
$$

$\ldots .$,

where $\beta$ is an angle between the $x$-axis and the projection of the fiber on the flow plane ( $x-y$ plane), and $\theta$ is an angle between the $z$-axis and the fiber.

\subsection{Solution procedure}

All governing equations were written in terms of non-dimensional variables using the channel width $H$ and the mean fluid velocity $U$ as the reference parameters. Subsequently, the Reynolds number was defined by $\mathrm{Re}=\rho U H / \eta$ and its value was set to 10 in the computations, where $\rho$ denotes the fluid density and $\eta$ the fluid viscosity. The computational domain was $x^{*}=0$ to 10 in the flow direction and $y^{*}=0$ to 0.5 in the width direction (the lower half part of the channel). The grid sizes were 0.025 in both the flow and the width directions.

The computations were carried out for mono- and bi-disperse suspensions. The computational conditions were chosen as $r_{a}=10$ in the mono-dispersion and 3 kinds of fiber aspect-ratio combinations in the bi-disperse case as shown in Table 1: $r_{a}=10$ and 20 (hereafter written by $\left.r_{a}=10+20\right), r_{a}=10+40$ and $r_{a}=10+100$. The fiber parameter $\phi \mu / \eta=\phi r_{a}^{2} / \ln \left(r_{a}\right)$ in Table 1 can be determined completely by the volume fraction and aspect-ratio of the fibers, $\phi$ and $r_{a}$, respectively. The total value of the fiber parameter was equal to 10 for these suspensions. Furthermore, the co-linear alignment of fibers on the wall was used as the simplest boundary condition for all the fiber aspect-ratios considered.

Table 1 Computational conditions for the mono- and bi-disperse fiber suspensions.

\begin{tabular}{c|c}
\hline condition & \multicolumn{1}{c}{$\begin{array}{c}\text { aspect-ratio of fibers: } \\
\text { fiber parameter }\end{array}$} \\
\hline mono-dispersion & $r_{a}=10: \phi \mu / \eta=10$ \\
bi-dispersion & $r_{a}=10+20, r_{a}=10+40$, \\
& $r_{a}=10+100:$ \\
& $\phi \mu / \eta=5$ for each aspect-ratio \\
& fiber \\
\hline
\end{tabular}


The computation time required for bi-disperse suspension flow was approximately 20 hours using a workstation with Alpha 21264 processor/750MHz.

We noticed the effect of number of fibers on the component of the fourth-order orientation tensor $a_{1111}$ for the 3-D orientation of mono-disperse suspensions (Azaiez et al. [15], see Fig. 27). Consequently, the appropriate number of segmentations $N_{\mathrm{s}}$ between 0 and $90^{\circ}$ in the $\theta$ direction were 30 or more. In the present computations of flow kinematics, $N_{\mathrm{s}}=30$ (the number of fibers $N=2,277$ for isotropic orientation state) for the mono-dispersion, while $N_{\mathrm{s}}$ was set to $18(N=816)$ for each aspect-ratio fiber of the bi-disperse suspensions in order to reduce the required $\mathrm{CPU}$ time, i.e., total number of fibers $N=1,632$ for the bi-disperse suspensions.

The evolution of the second-order orientation tensors depends significantly on the fiber aspect-ratios considered. In this work, the fiber orientation distribution in the simulated flows was estimated on the basis of the orientation angles of fibers whose number depended on the volume fraction and the fiber aspect-ratio, then the second-order orientation tensors were calculated from the orientation distribution of all fibers including different aspect-ratios. We calculated the number of fibers assuming the same fiber diameter for all the aspect-ratios considered.

\section{Flip-over and Oscillation Phenomena of the Orientation Ellipsoids}

We will discuss the mechanism of the flip-over or oscillation phenomenon of the orientation ellipsoid for mono- and bi-disperse suspensions. Detailed evolutions of the orientation ellipsoids within the region of their flip-over or oscillation phenomenon are shown in Fig.1. We can see clearly the evolutions of the projections of the orientation ellipsoids on both the $x-y$ plane (flow plane, Fig.1(a)) and the $x-z$ plane (Fig.1(b)) along the streamline passing the position of $y^{*}=1 / 40$ at the channel inlet, for mono- and bi-disperse suspensions.

We already reported [13] that fiber orientation distribution changed from isotropic distribution (drawn by a circle) at the channel inlet to co-linear alignment (straight line) in the downstream region for quasi infinite aspect-ratio suspension. On the other hand, the orientation ellipsoids frequently and periodically oscillated along the streamlines for suspensions including small aspect-ratio fibers irrespective of mono- and bi-disperse suspensions, and also the flip-over or oscillation phenomenon became significant as the channel wall was approached because of large velocity gradient existing in that region. It was also found that the period of these phenomena for the bi-disperse suspension completely depended on the period for the mono-dispersion involving the fiber with the smallest aspect-ratio ( $r_{a}=10$ in our case).

Fig.1(a) and (b) clearly show that the preferential direction of the orientation ellipsoids always lies on the $x-y$ plane even when the flip-over or oscillation phenomenon occurs owing to the isotropic orientation specified at the channel inlet. These figures illustrate the orientation distribution of all fibers including different aspect-ratios. On the other hand, the evolutions of the orientation ellipsoids for each aspect-ratio fiber of the bi-disperse suspensions are shown in Fig.2 (II, III, IV), which are the filled ellipses in Fig.1(a), in

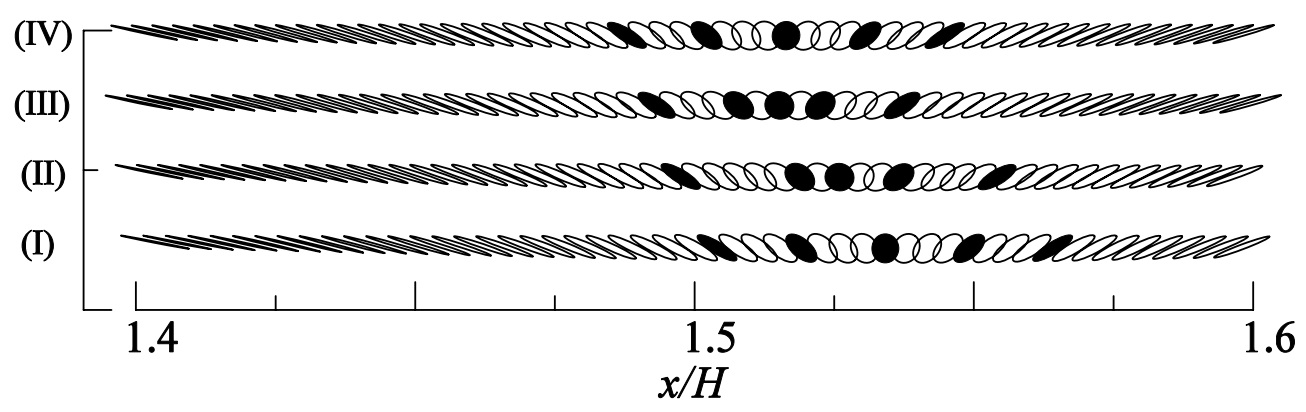

(a)

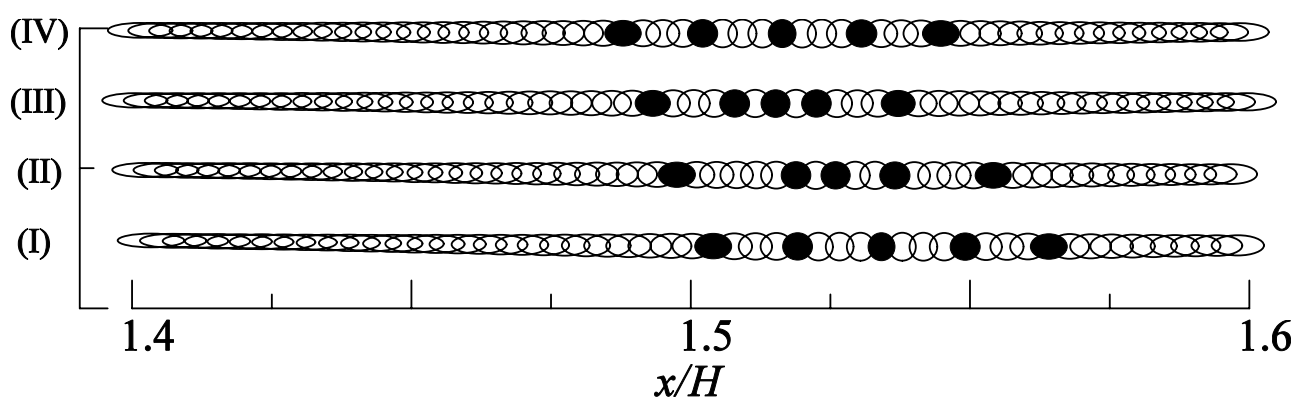

(b)

Fig.1 Evolution of the orientation ellipsoid within the region of flip-over or oscillation phenomenon for (I) $r_{a}=10$ mono-disperse suspension, (II) $r_{a}=10+20$, (III) $r_{a}=10+40$, (IV) $r_{a}=10+100$ bi-disperse suspensions; initial location is $y^{*}=1 / 40$; (a) projections of the orientation ellipsoids on the $x-y$ plane; (b) projections of the orientation ellipsoids on the $x-z$ plane. 


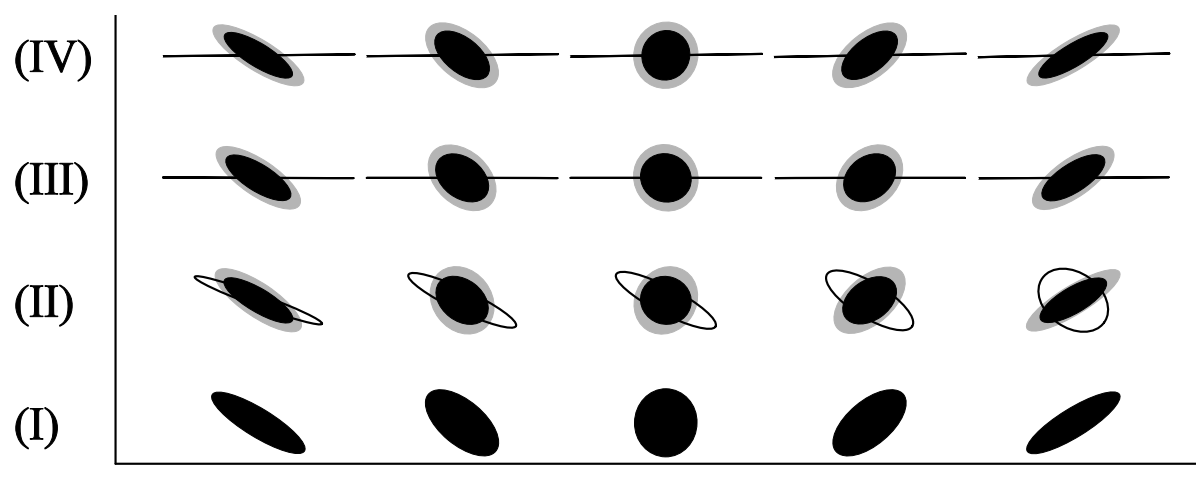

(a)

(b)

(c)

(d)

(e)

Fig.2 Detailed evolution of the orientation ellipsoid within the region of flip-over or oscillation phenomenon for (I) $r_{a}=10$ mono-disperse suspension, (II) $r_{a}=10+20$, (III) $r_{a}=10+40$, (IV) $r_{a}=10+100$ bi-disperse suspensions; initial location is $y^{*}=1 / 40$; black-filled ellipses express the orientation distributions for all suspended fibers, while grey-filled ellipses for small aspect-ratio fiber ( $r_{a}=10$ fiber) and unfilled ellipses for large aspect-ratio fiber.

order to discuss the mechanism of the flip-over or oscillation phenomenon in detail. These evolutions of the ellipsoids consist of grey filled ellipses for $r_{a}=10$ fiber, unfilled ellipses for large aspect-ratio fiber $\left(r_{a}=20,40\right.$ and 100) and filled ellipses for fibers including both aspect-ratios, where the filled ellipses are scaled by a factor of $2 / 3$ of the grey-filled and unfilled ellipses size for the sake of clarity. Fibers can almost completely align along the channel wall for fibers with $r_{a}=40$ and $r_{a}=100$ (see III and IV), however these fibers have little influence on the evolution of the orientation ellipsoids because the number of fibers with high aspect-ratios is much lower than the number of fibers with $r_{a}=10$ as shown in Table 2. In contrast it can be noticed that fibers with $r_{a}=20$ affect the evolution of the orientation ellipsoids in the bi-disperse suspensions (see II).

Figure 3 illustrates the variations of the second-order orientation tensor components and the preferential orientation of the ellipsoid for mono- and bi-disperse suspensions. Filled symbols in Fig. 3 correspond to the filled ellipses in Fig.1. The preferred angle abruptly changes from negative value to positive one for these three cases: it continuously changes through zero for the $r_{a}=10+20$ suspension (oscillation phenomenon), while it reaches \pm 90 degrees for the $r_{a}=10$ and the $r_{a}=10+100$ suspensions (flip-over phenomenon). The orientation ellipsoid oscillates for the $r_{a}=10+40$ suspension (not shown in Fig.3).

Table 2 Ratio of number of fibers for large aspect-ratios to that for $r_{a}=10$.

\begin{tabular}{c|c}
\hline aspect-ratio & ratio of number of fibers \\
\hline$r_{a}=10$ & 1.000 \\
$r_{a}=20$ & $1.626 \times 10^{-1}$ \\
$r_{a}=40$ & $2.503 \times 10^{-2}$ \\
$r_{a}=100$ & $2.000 \times 10^{-3}$ \\
\hline
\end{tabular}

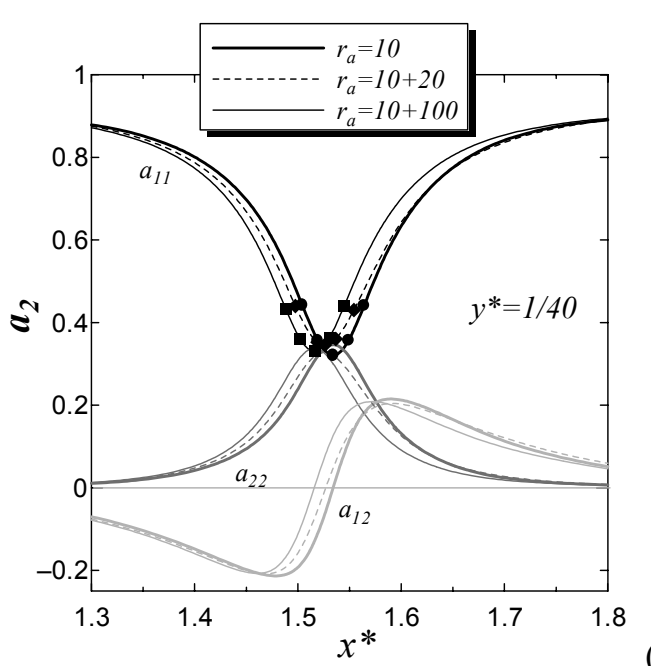

(a)

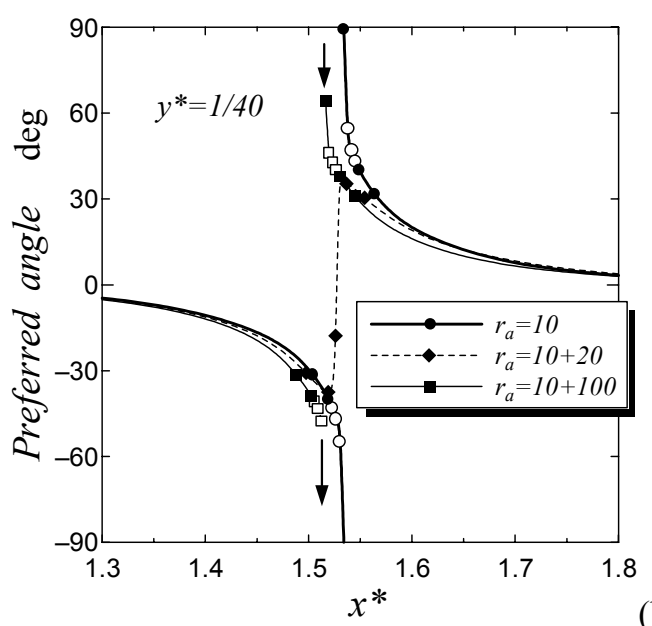

(b)

Fig.3 Variations of the 2nd-order orientation tensors and preferred angle of the orientation ellipsoid within the region of flip-over or oscillation phenomenon for $r_{a}=10$ mono-disperse suspension, $r_{a}=10+20$ and $r_{a}=10+100$ bi-disperse suspensions; initial location is $y^{*}=1 / 40$; (a) 2nd-order orientation tensor components; (b) preferred angle of the orientation ellipsoid. 


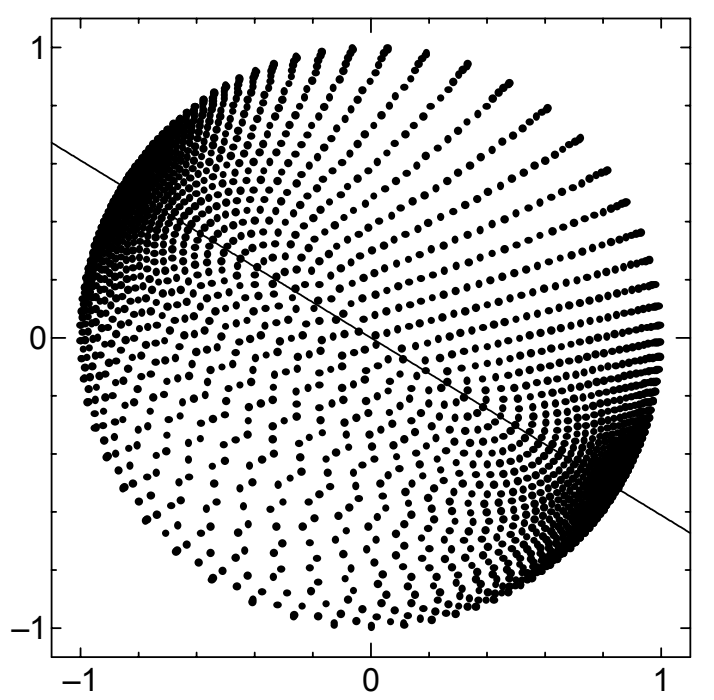

(a)

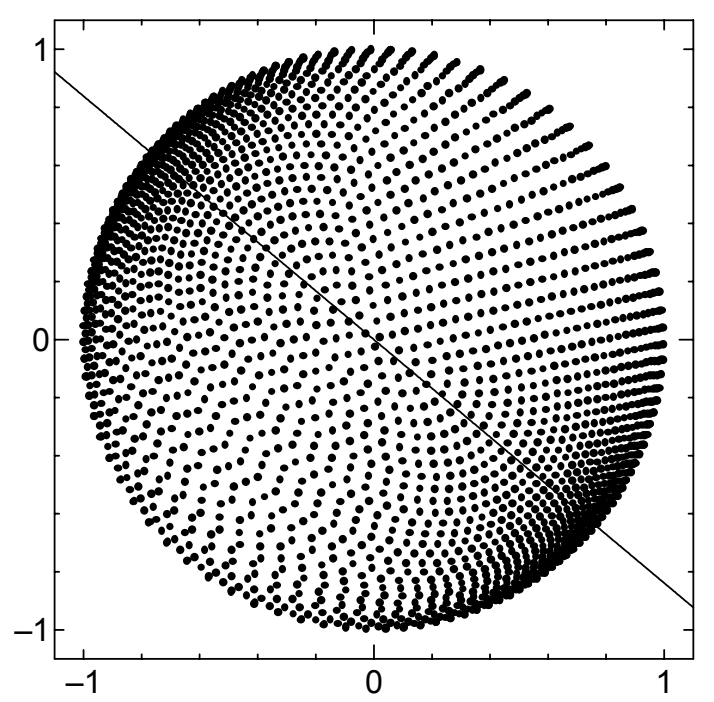

(b)

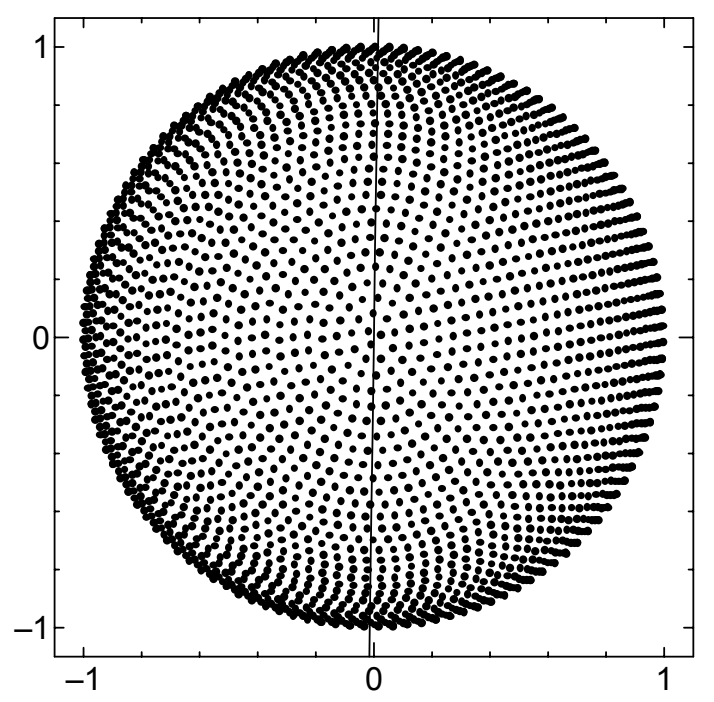

(c)

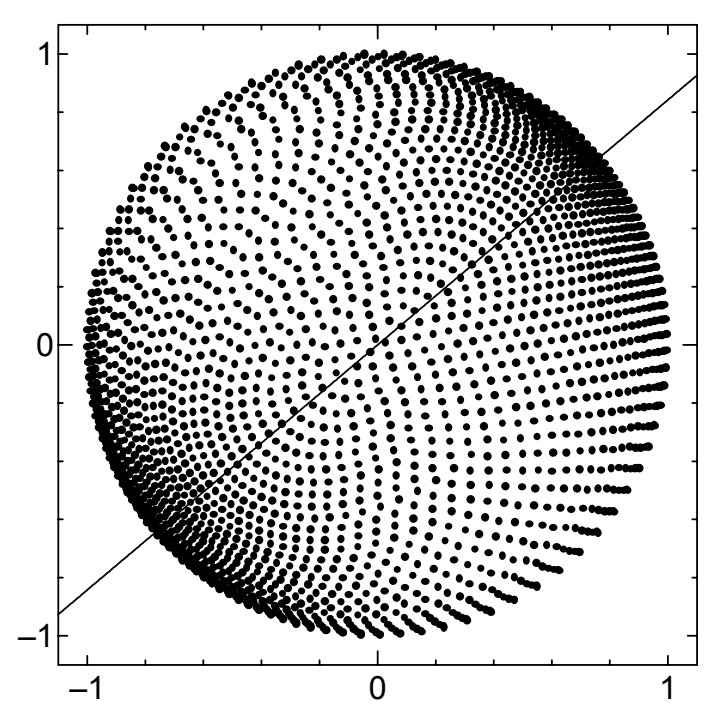

(d)

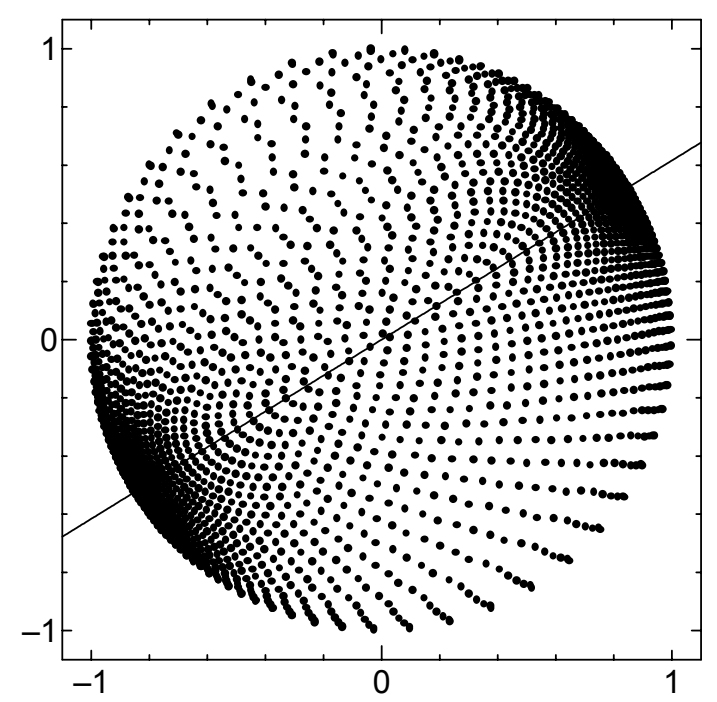

(e)

Fig.4 Transient evolution of the distribution of the orientation angle within the region of flip-over for $r_{a}=10$ mono-disperse suspension; initial location is $y^{*}=1 / 40$; preferred axis is drawn by a solid line;
(a) $x^{*}=1.504$, , (b) $x^{*}=1.519$, (c) $x^{*}=1.534$,
(d) $x^{*}=1.549$, (e) $x^{*}=1.564$. 


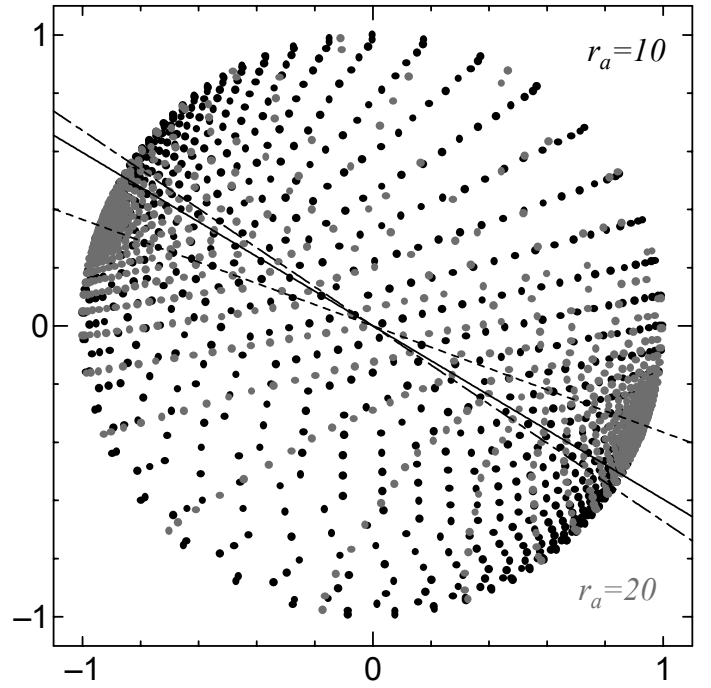

(a)

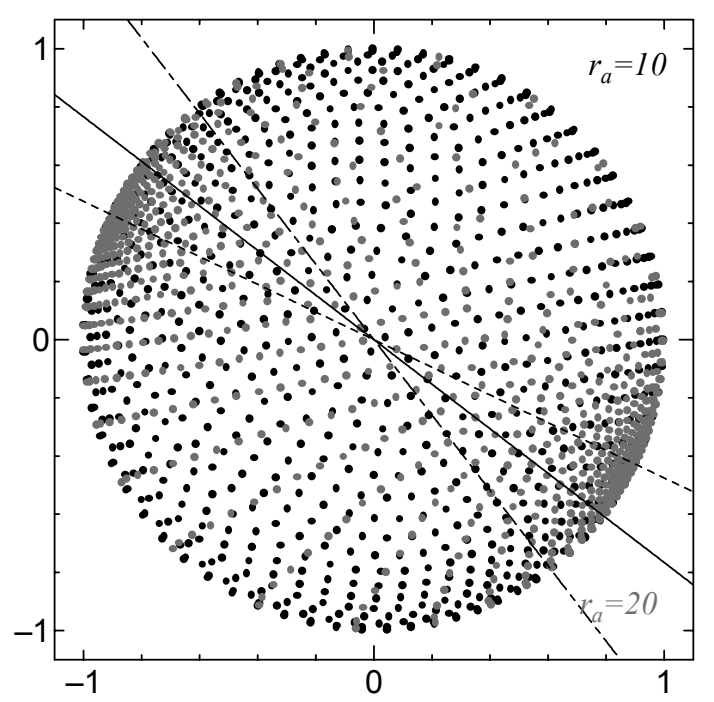

(b)

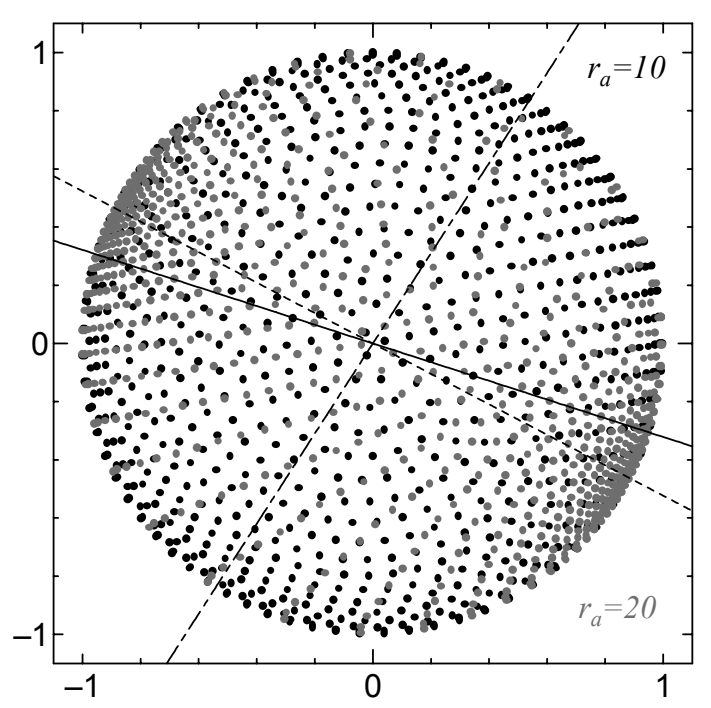

(c)

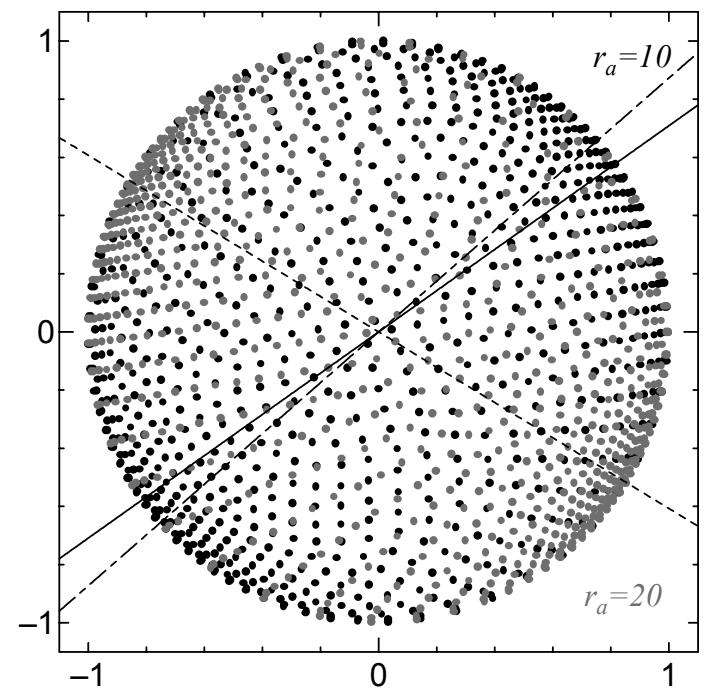

(d)

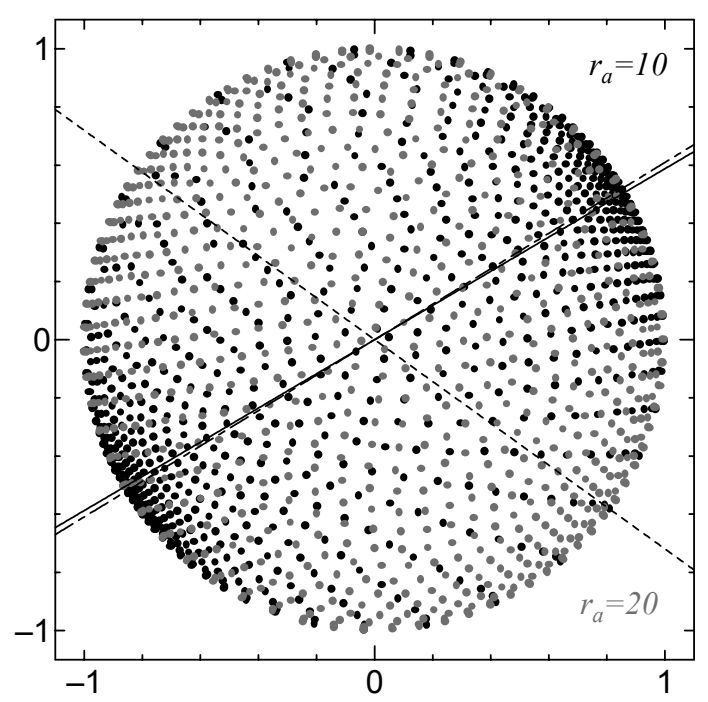

(e)

Fig.5 Transient evolution of the distribution of the orientation angle within the region of oscillation for $r_{a}=10+20$ bi-disperse suspension; initial location is $y^{*}=1 / 40 ;$ orientation distribution is illustrated by black-filled circle for $r_{a}=10$ and red-filled circle for $r_{a}=20$; preferred axes are drawn by a solid line for all fibers, by a dot-chain line for $r_{a}=10$ and by a broken line for $r_{a}=20$;
(a) $x *=1.498$
(b) $x^{*}=1.519$, (c) $x^{*}=1.526$
(d) $x^{*}=1.537$,
(e) $x^{*}=1.554$. 


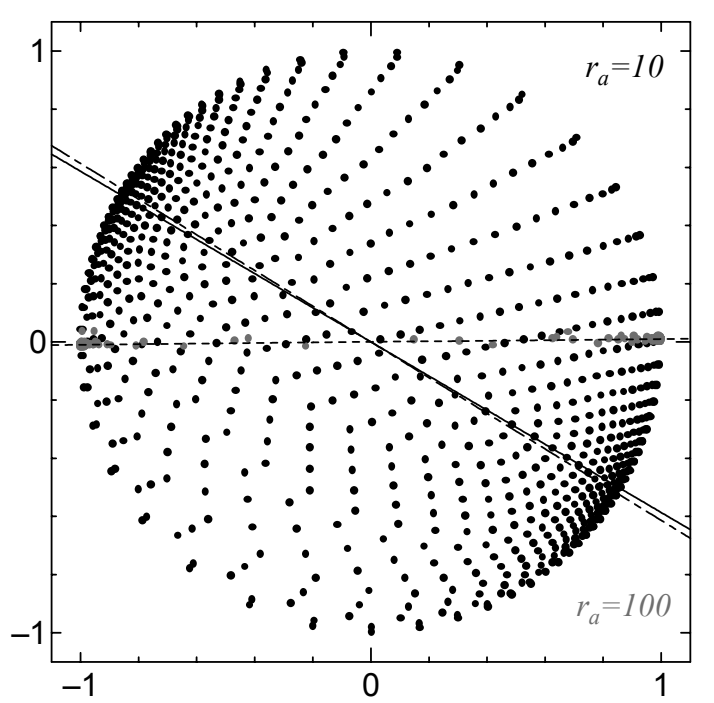

(a)

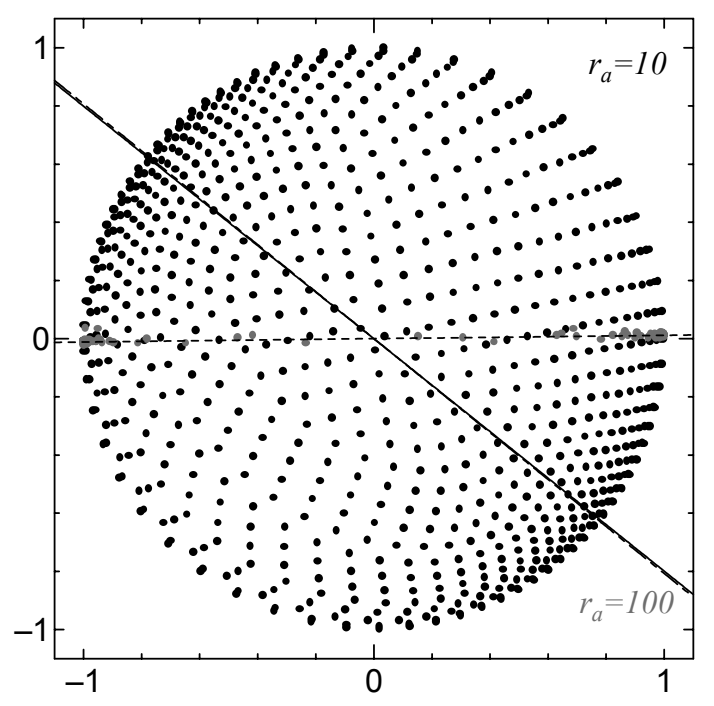

(b)

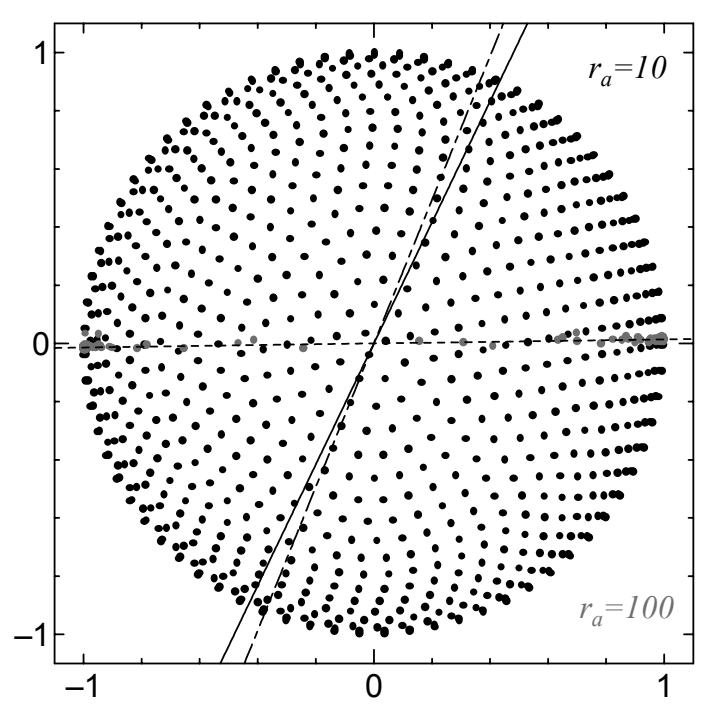

(c)

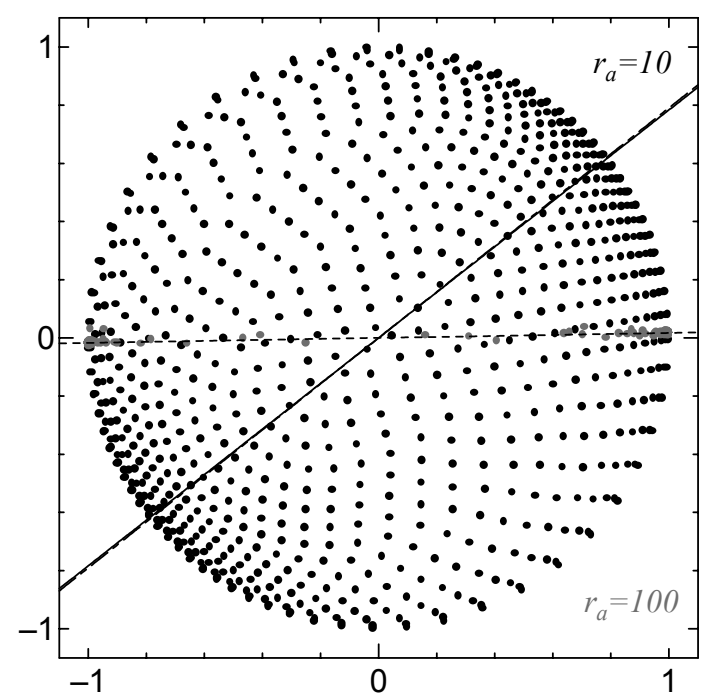

(d)

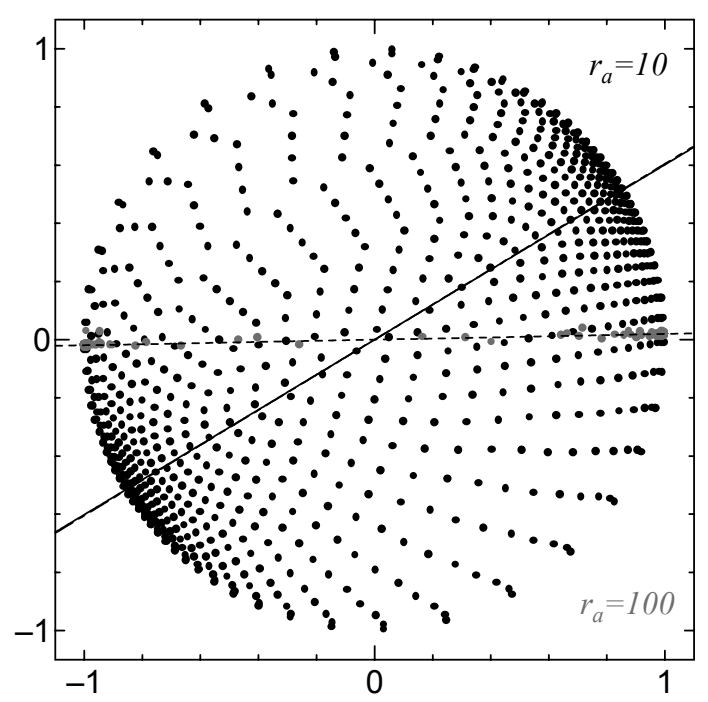

(e)

Fig.6 Transient evolution of the distribution of the orientation angle within the region of flip-over for $r_{a}=10+100$ bi-disperse suspension; initial location is $y^{*}=1 / 40$; orientation distribution is illustrated by black-filled circle for $r_{a}=10$ and red-filled circle for $r_{a}=100$; preferred axes are drawn by a solid line for all fibers, by a dot-chain line for $r_{a}=10$ and by a broken line for $r_{a}=100$;
(a) $x^{*}=1.488$,
(b) $x^{*}=1.502$, (c) $x^{*}=1.516$

(d) $x^{*}=1.531$, (e) $x^{*}=1.545$. 
On the other hand, $a_{11}$ becomes minimum, while $a_{22}$ maximum at the position of $a_{12}=0$, furthermore flip-over phenomenon occurs when the minimum value of $a_{11}$ becomes smaller than the maximum value of $a_{22}$, i.e., $a_{11}<1 / 3$. We can, therefore, confirm the above results of the flip-over or oscillation phenomenon from the variation of the second-order orientation tensor.

Finally, Figs.4 6 show the orientation distribution of fibers at the five positions drawn by filled ellipses and filled symbols in Figs. 1 and 3 for the $r_{a}=10, r_{a}=10+20$ and $r_{a}=10+100$ suspensions, respectively. These figures depict the projections of the one end of fibers on the $x-y$ plane, while the other end is located on the center of a unit sphere. For the $r_{a}=10$ mono-dispersion, the orientation distribution seems to be almost isotropic at the occurrence of flip-over (see Fig.4(c)), while fibers align around the preferred direction in Figs.4(a) and (e). However, the orientation distribution becomes a little asymmetric with the preferential direction. The orientation distribution for fibers with $r_{a}=20$ in the bi-dispersion with $r_{a}=10+20$ approaches from good alignment (Fig.5(a)) to almost isotropic state (Fig.5(e)). Furthermore, the occurrence of flip-over for $r_{a}=10$ does not coincide with the one associated with $r_{a}=20$, thus the orientation ellipsoid does not flip over but oscillates for the $r_{a}=10+20$ suspension. On the other hand, it can be seen in Fig. 6 that fibers with $r_{a}=100$ can almost completely align along the channel wall, however the preferential direction for the $r_{a}=10+100$ suspension (drawn by solid line) coincides with that for the $r_{a}=10$ fibers (dot-chain line), i.e., fibers with $r_{a}=100$ cannot affect the orientation distribution.

We were able to predict not only flip-over but also oscillation phenomena of the preferred axis in the evolution of the orientation distribution of a lot of fibers in a fluid element. This oscillation phenomenon of the preferred axis is similar to the wagging phenomenon of the director, which expresses the preferential direction of the molecular orientation distribution in a domain, in liquid crystalline flows. Furthermore, which phenomenon occurs, flip-over or oscillation, depends on the initial orientation distribution of fibers, the number of fibers of each aspect-ratio for bi-dispersion and the flow kinematics of fiber suspension.

The flip-over or oscillation phenomenon for multi-disperse suspensions occurs in a similar way to the above mechanism for bi-dispersions.

\section{Concluding Remarks}

Fiber suspension flow and fiber orientation through a parallel plate channel were numerically simulated for monoand bi-disperse suspensions using a statistical scheme. The statistical scheme was confirmed to be very useful and feasible method to analyze accurately the orientation distribution of fibers.

For suspensions including small aspect-ratio fibers, flip-over or oscillation phenomenon of the orientation ellipsoid was predicted. Which phenomenon occurs, flip-over or oscillation, depended on the initial orientation distribution of fibers and the number of fibers of each aspect-ratio for bi-dispersion as well as the flow kinematics of fiber suspension. Fibers with $r_{a}=40$ and $r_{a}=100$ almost completely aligned along the channel wall in the $r_{a}=10+40$ and $r_{a}=10+100$ suspension flows. Being compared with the number of fibers with $r_{a}=10$, the number of these large aspect-ratio fibers is much more reduced when we assumed the same fiber parameters for both small and large aspect-ratio fibers. For this reason these fibers had little influence on the evolution of the orientation ellipsoids. In contrast fibers with $r_{a}=20$ could significantly affect the evolution of the orientation ellipsoids in bi-disperse suspensions.

Fiber parameter $\phi \mu / \eta=10$ used in the present study corresponds to a semi-concentrated or concentrated regime of fiber suspension, thus the Jeffery equation is no more an appropriate way to describe the fiber orientation when fiber-fiber interaction is neglected. The effect of fiber-fiber interaction can be introduced in the Jeffery formalism from an appropriate Brownian term. We will address this question in a future work.

On the other hand, a still open problem which concerns the fiber suspension flows and that is encountered in the processing of composite materials of polymer matrix is the modeling of the role of the interaction between fibers and polymer molecules on the evolution of the fiber orientation.

\section{References}

[1]Lipscomb, G.G., Denn, M.M., Hur, D.H., Boger, D.V.; J. Non-Newtonian Fluid Mech., 26, 297 (1988)

[2]Chiba, K., Nakamura, K., Boger, D.V.; J. Non-Newtonian Fluid Mech., 35, 1 (1990)

[3]Rosenberg, J., Denn, M.M., Keunings, R.; J. Non-Newtonian Fluid Mech., 37, 317 (1990)

[4]Ausias, G., Agassant, J.F., Vincent, M., Lafleur, P.G., Lavoie, P.A., Carreau, P.J.; J. Rheol., 36, 525 (1992)

[5]Ausias, G., Agassant, J.F., Vincent, M.; Intern. Polym. Proc., 9, 51 (1994)

[6]Ghosh, T., Grmela, M., Carreau, P.J.; Polym. Compos., 16, 144 (1995)

[7]Azaiez, J., Guenette, R., Ait-Kadi, A.; J. Non-Newtonian Fluid Mech., 73, 289 (1997)

[8]Zirnsak, M.A., Boger, D.V.; J. Non-Newtonian Fluid Mech., 79, 105 (1998)

[9]Fan, X.-J., Phan-Thien, N., Zheng, R.; J. Non-Newtonian Fluid Mech., 84, 257 (1999)

[10]Chung, D.H., Kwon, T.H.; J. Non-Newtonian Fluid Mech., 107, 67 (2002)

[11]Chiba, K., Chinesta, F.; Mise en forme des matériaux composites, Revue des composites et des matériaux avancés, 12, 499 (2002)

[12]Shiraishi, Y., Narazaki, N., Kikutani, T.; Polym. Composites, 25, 194 (2004)

[13]Chiba, K., Chinesta, F.; Rheol. Acta, in press

[14]Advani, S.G., Tucker III, C.L.; J. Rheol., 31, 751 (1987)

[15]Azaiez, J., Chiba, K., Chinesta, F., Poitou, A.; Arch. Comput. Meth. Engng., 9, 141 (2002) 